\title{
THE QCD ANALYTIC EFFECTIVE CHARGE AND ITS DEPENDENCE ON THE PION MASS
}

\author{
A.V. NESTERENKO ${ }^{1}$ and J. PAPAVASSILIOU ${ }^{2}$ \\ ${ }^{1}$ Bogoliubov Laboratory of Theoretical Physics, Joint Institute for Nuclear Research \\ Dubna, 141980, Russian Federation \\ ${ }^{2}$ Departamento de Física Teórica and IFIC, Centro Mixto, \\ Universidad de Valencia-CSIC, E-46100, Burjassot, Valencia, Spain
}

Received 18 September 2004

\begin{abstract}
A new model for the QCD analytic running coupling, which incorporates the effects due to the $\pi$ meson mass, is proposed. The properties of this invariant charge in spacelike and timelike regions are examined. Its main distinctive features are a finite infrared limiting value, which depends on the pion mass, and the "plateau-like" behavior in the deep infrared domain of the timelike region.
\end{abstract}

Keywords: Nonperturbative QCD; dispersion relations; strong running coupling.

\section{Introduction}

The description of the infrared hadron dynamics remains a crucial challenge for elementary particle physics. Whereas perturbative calculations allow for a detailed study of the strong interaction processes at high energies, to date there is no reliable theoretical method which would enable one to handle the hadron dynamics at low energies. However, many physical phenomena are tightly bound to the intrinsically nonperturbative aspects of the strong interaction.

The renormalization group (RG) method plays a fundamental role in the framework of Quantum Field Theory (QFT) and its applications. Usually, in order to describe Quantum Chromodynamics (QCD) in the ultraviolet region, one applies the RG method together with perturbative calculations. Eventually, this leads to approximate solutions of the RG equations, which are commonly used in the quantitative analysis of high energy processes. However, such solutions possess unphysical singularities in the infrared domain, a fact that contradicts the general principles of local QFT and complicates the theoretical description of the intermediate and low energy experimental data. Nevertheless, these difficulties can be avoided if one complements the perturbative results with a proper nonperturbative insight.

For this purpose the relevant dispersion relations can be used. The idea of employing the information "stored" in such integral representations together with per- 
turbation theory forms the basis of the analytic approach to QFT. First it was proposed in the framework of Quantum Electrodynamics $\$$ and lately it was extended 2 to QCD. Another important application of the dispersion relations is the analysis of the timelike QCD experimental data. Here, the dispersion relation for the Adler $D$ function 3 enables one to handle the timelike strong interaction processes in a consistent way.

The effects due to the masses of the lightest hadrons can be safely neglected only when one studies the QCD processes at high energies. But for the description of the low energy dynamics the mass effects become essential. For example, this is crucial for the description of the inclusive $\tau$ lepton decay. Both, the perturbative results and the dispersion relation for the Adler $D$ function with the nonvanishing pion mass, are essential here for the correct interpretation of these data. However, no such mass effects have been taken into account in the analytic approach to QCD so far.

In this talk we report recent progress 4 on the incorporation of the effects due to the $\pi$ meson mass into the analytic approach to QCD, and the study of the behavior of the developed running coupling in spacelike and timelike infrared domains.

\section{Strong Running Coupling in Spacelike and Timelike Regions}

The description of hadron dynamics in spacelike and timelike regions has been the subject of many studies over a long period of time. The strong interaction processes with the large spacelike momentum transfer $q^{2}>0$ can be examined perturbatively in the framework of the RG method (the metric with the signature $(-1,1,1,1)$ is used, so that positive $q^{2}$ corresponds to a spacelike momentum transfer). But in order to handle the processes in the timelike region $\left(s=-q^{2}>0\right)$, one has first to relate the perturbative results with the measured quantities.

A substantial step towards the consistent description of the timelike data was made in Refs. 3] [5] and 6] Namely, it has been argued that the dispersion relation for the Adler $D$ function

$$
D\left(q^{2}\right)=q^{2} \int_{4 m_{\pi}^{2}}^{\infty} \frac{R(s)}{\left(s+q^{2}\right)^{2}} d s
$$

supplies a firm ground for comparing the perturbative results for $D\left(q^{2}\right)$ with the experimental measurements of the $R(s)$-ratio of the $e^{+} e^{-}$annihilation into hadrons. Thus, the perturbative results for $D\left(q^{2}\right)$ can be continued into the timelike domain by making use of the relation inverse to (10) (see also Refs. [5] and 7)

$$
R(s)=\frac{1}{2 \pi i} \lim _{\varepsilon \rightarrow 0_{+}} \int_{s+i \varepsilon}^{s-i \varepsilon} D(-\zeta) \frac{d \zeta}{\zeta} .
$$

The asymptotic ultraviolet behavior of the Adler $D$ function can be computed by making use of the perturbation theory 78

$$
D\left(q^{2}\right)=N_{\mathrm{c}} \sum_{f} Q_{f}^{2}\left[1+d\left(q^{2}\right)\right], \quad d\left(q^{2}\right) \simeq d_{1}\left[\frac{\alpha_{\mathrm{s}}\left(q^{2}\right)}{\pi}\right]+d_{2}\left[\frac{\alpha_{\mathrm{s}}\left(q^{2}\right)}{\pi}\right]^{2}+\cdots
$$


Here $d_{1}=1, d_{2} \simeq 1.9857-0.1153 n_{\mathrm{f}}, n_{\mathrm{f}}$ is the number of active quarks, $N_{\mathrm{c}}=3$ is the number of colors, and $Q_{f}$ stands for the charge of the $f$-th quark. Since the integral transformation (2) of Eq. (3) has to be performed every time when one deals with the timelike experimental data, it is convenient to define ${ }^{9}$ the timelike effective charge $\widehat{\alpha}(s)$ in the same way, as $R(s)$ relates to $D\left(q^{2}\right)$ :

$$
\widehat{\alpha}(s)=\frac{1}{2 \pi i} \lim _{\varepsilon \rightarrow 0_{+}} \int_{s+i \varepsilon}^{s-i \varepsilon} \alpha(-\zeta) \frac{d \zeta}{\zeta} .
$$

Here and further the strong running coupling in the spacelike and timelike domains is denoted by $\alpha\left(q^{2}\right)$ and $\widehat{\alpha}(s)$, respectively. The inverse relation

$$
\alpha\left(q^{2}\right)=q^{2} \int_{4 m_{\pi}^{2}}^{\infty} \frac{\widehat{\alpha}(s)}{\left(s+q^{2}\right)^{2}} d s
$$

holds as well. Evidently, for the thorough description of the QCD processes at low energies the pion mass cannot be neglected in Eqs. (11) and (15).

For the self-consistency of the method described above, one has first to bring the perturbative approximation for the Adler $D$ function (3) to conformity with the dispersion relation (11). Indeed, Eq. (11) implies that in the massless case $\left(m_{\pi}=0\right)$ $D\left(q^{2}\right)$ is the analytic function in the complex $q^{2}$-plane with the only cut $-\infty<q^{2} \leq$ 0 along the negative semiaxis of real $q^{2}$. However, the perturbative approximation of $d\left(q^{2}\right)$ in Eq. (3) violates this condition. Fortunately, this discrepancy can be avoided in the framework of the analytic approach to QCD.

\section{Massless Analytic Effective Charge}

As was mentioned in the Introduction, the dispersion relations provide one with a certain nonperturbative information about a quantity in hand, namely, with the definite analytic properties in the kinematic variable. Recently it was argued 2 that in the massless case the Källén-Lehmann spectral representation for the QCD invariant charge

$$
\alpha\left(q^{2}\right)=\int_{0}^{\infty} \frac{\varrho(\sigma)}{\sigma+q^{2}} d \sigma
$$

holds. It is precisely this condition that is needed in order to bring the perturbative approximation for the Adler $D$ function (3) in agreement with the dispersion relation (1) and to satisfy the relations (4) and (5) in the limit of the massless pion $m_{\pi}=0$.

This section is devoted to a brief overview of one of the massless models for the QCD analytic running coupling 10 The distinguishing feature of this model is that the analyticity requirement is imposed on the perturbative expansion of the $\beta$ function. This way of incorporating the analyticity requirement into the $\mathrm{RG}$ formalism is consistent with the general definition of the QCD invariant charge.1112 The mode 10 has proved to be successful in the description of the hadron dynamics 
of the both perturbative and intrinsically nonperturbative nature 13 The running coupling 10 has also been rederived in Ref. 14 proceeding from different assumptions.

Thus, the Källén-Lehmann representation (6) holds for the massless analytic invariant charge (the relevant details can be found in Refs. 10 and 11)

$$
\alpha_{\mathrm{an}}^{(\ell)}\left(q^{2}\right)=\frac{4 \pi}{\beta_{0}} \int_{0}^{\infty} \frac{\rho^{(\ell)}(\sigma)}{\sigma+z} d \sigma, \quad z=\frac{q^{2}}{\Lambda^{2}} .
$$

In this equation $\rho^{(\ell)}(\sigma)$ denotes the $\ell$-loop spectral density

$$
\rho^{(\ell)}(\sigma)=\rho^{(1)}(\sigma) \exp \left[f_{0}^{\infty} \mathcal{P}^{(\ell)}(\zeta) \ln \left|1-\frac{\zeta}{\sigma}\right| \frac{d \zeta}{\zeta}\right]\left[\cos \psi^{(\ell)}(\sigma)+\frac{\ln \sigma}{\pi} \sin \psi^{(\ell)}(\sigma)\right],
$$

where $\rho^{(1)}(\sigma)=\left(1+\sigma^{-1}\right) /\left(\ln ^{2} \sigma+\pi^{2}\right)$ stands for the one-loop spectral density, $\psi^{(\ell)}(\sigma)=\pi \int_{\sigma}^{\infty} \mathcal{P}^{(\ell)}(\zeta) \zeta^{-1} d \zeta, \mathcal{P}^{(\ell)}(\sigma)=\mathcal{R}^{(\ell)}(\sigma)-\mathcal{R}^{(1)}(\sigma)$, and

$$
\mathcal{R}^{(\ell)}(\sigma)=\frac{1}{2 \pi i} \lim _{\varepsilon \rightarrow 0_{+}} \sum_{j=0}^{\ell-1} \frac{\beta_{j}}{(4 \pi)^{j+1}}\left\{\left[\alpha_{\mathrm{s}}^{(\ell)}(-\sigma-i \varepsilon)\right]^{j+1}-\left[\alpha_{\mathrm{s}}^{(\ell)}(-\sigma+i \varepsilon)\right]^{j+1}\right\} .
$$

Here $\beta_{0}=11-2 n_{\mathrm{f}} / 3, \beta_{1}=102-38 n_{\mathrm{f}} / 3, \alpha_{\mathrm{s}}^{(\ell)}\left(q^{2}\right)$ is the $\ell$-loop perturbative running coupling. In the exponent in Eq. (8) the principal value of the integral is assumed.

The model (7) shares all the advantages of the analytic approach: it contains no unphysical singularities, displays good higher-loop and scheme stability, and has no adjustable parameters. The massless analytic effective charge (7) incorporates the ultraviolet asymptotic freedom with the infrared enhancement in a single expression, that plays a crucial part when applying this model to the study of quenched lattice simulation data. 14 The detailed analysis of the properties of the invariant charge (7) and its applications can be found in Refs. $10-13$

For the congruous description of the timelike hadron dynamics one has to employ the continuation (4) of the strong running coupling. This leads to the following extension of the invariant charge (7) to the timelike domain 10 (see also Ref. 9):

$$
\widehat{\alpha}_{\mathrm{an}}^{(\ell)}(s)=\frac{4 \pi}{\beta_{0}} \int_{w}^{\infty} \rho^{(\ell)}(\sigma) \frac{d \sigma}{\sigma}, \quad w=\frac{s}{\Lambda^{2}},
$$

where $s=-q^{2}>0$, and the spectral density $\rho^{(\ell)}(\sigma)$ is defined in Eq. (8). The plots of the functions $\alpha_{\mathrm{an}}^{(1)}\left(q^{2}\right)$ and $\widehat{\alpha}_{\mathrm{an}}^{(1)}(s)$ are shown in Figure 1. Note the asymmetry between these curves in the intermediate and low energy regions, which has to be taken into account when one handles the experimental data (see review 11 and references therein for the details).

It is worth noting that the formulation of the analytic approach to QCD is still essentially massless. The obtained results can be applied, for example, to the study of the experimental data at high energies, the pure gluodynamics, and the quenched lattice simulation data. However, for a consistent description of the infrared hadron dynamics, the mass effects have to be incorporated into the analytic approach to QCD. 


\section{Massive Analytic Effective Charge}

The original dispersion relation for the Adler $D$ function 3 (11) with the nonvanishing mass of the $\pi$ meson implies that $D\left(q^{2}\right)$ is the analytic function in the complex $q^{2}$-plane with the only cut beginning at the two-pion threshold $-\infty<q^{2} \leq-4 m_{\pi}^{2}$. However, the approximation (3) violates this condition due to the spurious singularities of the perturbative running coupling. This disagreement can be avoided by imposing the analyticity requirement of the form $d\left(q^{2}, m_{\pi}^{2}\right)=\int_{4 m_{\pi}^{2}}^{\infty} \varkappa(\sigma)\left(\sigma+q^{2}\right)^{-1} d \sigma$ on the function $d\left(q^{2}\right)$ in Eq. (31). Thus, the strong running coupling itself has to satisfy the integral representation

$$
\alpha\left(q^{2}, m_{\pi}^{2}\right)=\int_{4 m_{\pi}^{2}}^{\infty} \frac{\varrho(\sigma)}{\sigma+q^{2}} d \sigma .
$$

Otherwise, one would encounter the contradiction between the dispersion relation for the Adler $D$ function (11) and its approximation (3).

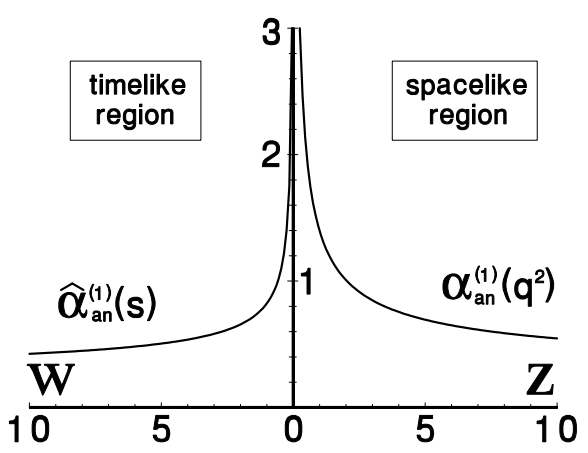

Fig. 1. The one-loop massless analytic running coupling in spacelike (Eq. [7), $q^{2}>0$ ) and timelike (Eq. 10], $s=-q^{2}>0$ ) regions, $z=-w=q^{2} / \Lambda^{2}$

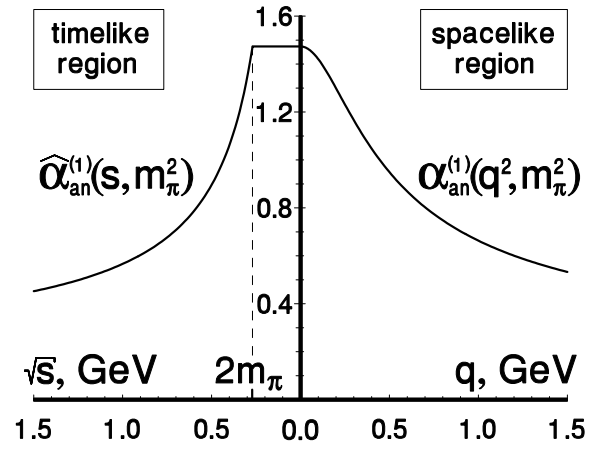

Fig. 2. The one-loop massive analytic running coupling in spacelike (Eq. [12) and timelike (Eq. 13) domains. The value $\Lambda=$ $623 \mathrm{MeV}$ and two active quarks are assumed.

There are several ways to restore the spectral density $\varrho(\sigma)$ in the integral representations (6) and (11). Ultimately, this has led to different models for the strong running coupling within the analytic approach to QCD (see Refs. 10,12, 15). The model for the analytic invariant charge ${ }^{10}$ has proved to be successful in the description of both perturbative and intrinsically nonperturbative strong interaction processes. 1113 We will therefore adopt in this work the spectral density (8).

Thus, one arrives at the following integral representation for the massive analytic effective charge

$$
\alpha_{\mathrm{an}}^{(\ell)}\left(q^{2}, m_{\pi}^{2}\right)=\frac{4 \pi}{\beta_{0}} \int_{\chi}^{\infty} \frac{\rho^{(\ell)}(\sigma)}{\sigma+z} d \sigma, \quad z=\frac{q^{2}}{\Lambda^{2}},
$$

where $\rho^{(\ell)}(\sigma)$ denotes the $\ell$-loop spectral density (8), and $\chi=4 m_{\pi}^{2} / \Lambda^{2}$. It turns out that the nonvanishing mass of the $\pi$ meson drastically affects the infrared behavior 
of the running coupling. Indeed, instead of the infrared enhancement in the massless case (7), one has here the infrared finite limiting value for the effective charge (12) $\alpha_{0}^{(\ell)}(\chi)=4 \pi \beta_{0}^{-1} \int_{\chi}^{\infty} \rho^{(\ell)}(\sigma) \sigma^{-1} d \sigma$. The continuation (4) of the running coupling (12) results in the following representation for the timelike massive effective charge:

$$
\widehat{\alpha}_{\mathrm{an}}^{(\ell)}\left(s, m_{\pi}^{2}\right)=\frac{4 \pi}{\beta_{0}} \int_{w}^{\infty} \theta(\sigma-\chi) \rho^{(\ell)}(\sigma) \frac{d \sigma}{\sigma}, \quad w=\frac{s}{\Lambda^{2}},
$$

where $s=-q^{2} \geq 0, \rho^{(\ell)}(\sigma)$ is the $\ell$-loop spectral density (8), and $\chi=4 m_{\pi}^{2} / \Lambda^{2}$.

The effective charges (12) and (13) have a common finite value $\alpha_{0}^{(\ell)}(\chi)$ in the infrared limit. Then, the timelike coupling (13) has the "plateau" in the infrared domain: $\widehat{\alpha}_{\text {an }}^{(\ell)}\left(s, m_{\pi}^{2}\right)=\alpha_{0}^{(\ell)}(\chi)$, when $0 \leq \sqrt{s} \leq 2 m_{\pi}$ (see Fig. 2). The massless (10) and massive (13) timelike couplings coincide for $\sqrt{s}>2 m_{\pi}$, since the pion mass affects the coupling (13) only in the region $0 \leq \sqrt{s} \leq 2 m_{\pi}$, where it stops running.

Finally, the developed model has been applied 4 to the study of the experimental data on the inclusive $\tau$ lepton decay, 16 that has given (at the one-loop level with two active quarks) the value $\Lambda=(623 \pm 81) \mathrm{MeV}$ of the QCD scale parameter.

\section{Acknowledgments}

A.N. is grateful to I.L. Solovtsov for valuable discussions and comments. Support by grants RFBR (02-01-00601, 04-02-81025), NS-2339.2003.2, and CICYT FPA2000200612 is acknowledged.

\section{References}

1. P.J. Redmond, Phys. Rev. 112, 1404 (1958); N.N. Bogoliubov, A.A. Logunov, and D.V. Shirkov, Sov. Phys. JETP 37, 574 (1960).

2. D.V. Shirkov and I.L. Solovtsov, Phys. Rev. Lett. 79, 1209 (1997).

3. S.L. Adler, Phys. Rev. D10, 3714 (1974).

4. A.V. Nesterenko and J. Papavassiliou, in preparation.

5. A.V. Radyushkin, JINR Report No. 2-82-159, 1982; arXiv:hep-ph/9907228

6. N.V. Krasnikov and A.A. Pivovarov, Phys. Lett. B116, 168 (1982).

7. S.G. Gorishny, A.L. Kataev, and S.A. Larin, Phys. Lett. B259, 144 (1991).

8. L.R. Surguladze and M.A. Samuel, Phys. Rev. Lett. 66, 560 (1991).

9. K.A. Milton and I.L. Solovtsov, Phys. Rev. D55, 5295 (1997); D59, 107701 (1999).

10. A.V. Nesterenko, Phys. Rev. D62, 094028 (2000); D64, 116009 (2001).

11. A.V. Nesterenko, Int. J. Mod. Phys. A18, 5475 (2003).

12. A.V. Nesterenko and I.L. Solovtsov, Mod. Phys. Lett. A16, 2517 (2001).

13. A.V. Nesterenko, Nucl. Phys. (Proc. Suppl.) B133, 59 (2004).

14. F. Schrempp, J. Phys. G28, 915 (2002).

15. D.V. Shirkov, Theor. Math. Phys. 132, 1309 (2002); arXiv:hep-ph/0408272

16. R. Barate et al. (ALEPH Collaboration), Eur. Phys. J. C4, 409 (1998);

K. Ackerstaff et al. (OPAL Collaboration), ibid. C7, 571 (1999). 